\title{
BRINGING PERSONAS TO LIFE: USER EXPERIENCE DESIGN THROUGH INTERACTIVE COUPLED OPEN INNOVATION
}

\author{
LyNn CoOREVITs, Dimitri SchuURMAN, \\ KATHY OELBRANDT, AND SARA LOGghe
}

\begin{abstract}
A frequently used technique to enable user involvement during the course of a software design project is the development of personas. Modeling personas helps developers to establish a stronger user focus and act as a constant reminder for whom one is and is not designing. Within this paper we propose coupled interactive open innovation through iterative user involvement, such as in a Living Lab approach, as a way to improve the effectiveness of personas and scenarios. Interactive coupled open innovation addresses user needs and creates new opportunities by using a trial-error learning process. This increases efficiency, augments stakeholders' collaboration and facilitates co-creation. By involving real users who mirror the constructed personas in subsequent development steps, traditional persona development is enriched. This iterative process elicits new understandings and meanings of domain specific and market knowledge by positioning the development project in a 'real-life' context. The input of each step in the process translates towards and provides the technical team with potential input to develop iterations of the design. In this paper we provide a framework, based on interactive coupled open innovation, for persona development that will allow an optimized user experience by bringing personas and their scenarios to life in the design project.
\end{abstract}

\section{KEY WORDS}

Interactive Coupled Open Innovation; User Experience; Persona; Innovation; Living Lab methodology

\section{INTRODUCTION}

The front end of design in new software development is a critical phase in which careful consideration of the decisions is necessary (Sanders and Stappers 3). Personas and their scenarios can play an important role in design orientation, especially when the project consists of a large team of developers or a diverse set of users (Matthews, Judge, and Whittaker 1219). The scenarios predict how certain types of users, represented by personas, will interact with the system in a given situation in order to complete a certain goal. This enables teams to understand user needs, and shifts the focus towards the accompanying software requirements which makes communication to the different stakeholders in the project easier (Markensten and Artman 14). 
The added value of personas has been demonstrated in various settings, but strategies for effectively using personas diverge in several ways (Pruitt and Grudin 314). Researchers and practitioners find the creation process challenging and the existing guidelines are open to interpretation (Matthews, Judge, and Whittaker 1219). Furthermore effective use remains elusive because the persona is often perceived as too abstract, the information as irrelevant and underlying data as untrustworthy. Matthews, Judge and Wittaker suggest that if persona development is linked to user study data it could improve their effectiveness by helping developers to decide upon "critical" information for the design (1227). Also Chapman and Milham as well as Pruitt and Grudin argue the importance of reconciling personas and their scenarios with other data. To accomplish this, Pruitt and Grudin suggest that a combination of qualitative and quantitative user data can provide the underlying information needed for persona creation. This in turn will make them less subjective and more credible for developers because they can verify how they are being constructed (324). There is some disagreement however on how to create personas and which approach would be most useful to developers (Matthews, Judge, and Whittaker 1221).

Within this paper, we will integrate an "interactive coupled open innovation approach" (Piller and West) to support the development of personas. It is assumed that user-driven methodologies, such as a Living Lab methodology, can enhance the outcomes of design methods because personas in themselves cannot provide input on "product answers relating to usability and usefulness" (Dotan et al. 3). Adopting a Living Lab methodology should "blur the lines between technology users and producers by including (future) users of technologies from the start of technology development" (Sauer 16). Researchers using this methodology can mediate the innovation process, "by aligning the different stakeholders and articulating product characteristics and requirements" (Coenen and Robijt 1). The "iterative nature" of this type of persona development allows for trial and error and the introduction of variations that produce different results (Trimi and Berbegal-Mirabent 459). In the literature there are currently no studies available that have combined persona creation with an interactive coupled open innovation approach. Therefore this paper will examine by means of a single case study how iterative immersion in user data and working with real end-users, in a design project via the Living Lab methodology, can improve the effective usage and outcomes of applying personas in user experience research and design.

\section{PERSONAS}

In user experience research, personas are an established "interaction design technique" for software applications (Pruitt and Grudin 313) because they help developers decide upon product requirements, interaction patterns, and presentational design. Personas are fictional characters that embody typical characteristics of the different (potential) user groups of the innovation under development. They are a good starting point for user experience design work (Johansson and Messeter; Floyd, Cameron Jones, and Twidale), because they explain the end users' "attitudes towards technology" in relation to their daily life (Dotan et al. 4), which makes the persona "more vivid" and "memorable" (Floyd, Cameron Jones, and Twidale 8). Their accompanying scenarios explain how personas would interact with the new system, eliciting key requirements for the developers (Long). Envisioning real users in the form of a persona rather than abstract groups of people makes it easier for developers to focus on the user. Personas have also proved their effectiveness as a communication medium between project participants (Grudin and Pruitt 326). Despite these promising features, the designer's' willingness to use personas in practice varies tremendously (Matthews, Judge, and Whittaker). Sometimes they are considered as too subjective (Floyd, Cameron Jones, and Twidale), "abstract", and "impersonal" (Matthews, Judge, and Whittaker 1220). Additionally it is too 
difficult for developers to verify whether and how well they reflect user data (Dotan et al. 4). Even experienced user centered design practitioners are confronted with challenges while using personas (Matthews, Judge, and Whittaker 1226). In order to deal with those challenges it is critical to "immerse in user data" to make more accurate reflections of the user population (Matthews, Judge, and Whittaker 1227). In other words personas can no longer be seen as a standalone technique to understand users and their needs. Interactive coupled open innovation such as the Living Lab methodology can provide structure to this process by performing user research and bringing personas to life as real users or participants in the development process.

\section{INTERACTIVE COUPLED OPEN INNOVATION}

Piller and West suggest "interactive coupled open innovation" as a model where organizations and users jointly create "new knowledge and input for the innovation process" (39). This process consists of the following stages: defining, finding participants, collaborating, and leveraging. Piller and West regard co-creation as the dominant activity during this process and see this as a link between both the Open and User Innovation paradigms. Nevertheless, they also notice a lack of research into co-creation as a process and a lack of management frameworks for implementing interactive coupled open innovation.

Within this paper, we perceive Living Labs as multi-facetted phenomena embodying both Open and User Innovation. Building further on the argument of Piller and West, Schuurman suggests Living Labs as "infrastructures facilitating and enabling interactive coupled open innovation" (319). Living Labs function as "experimental platforms" where end-users are studied in their everyday context (Eriksson, Niitamo, and Kulkki 7). However, in the literature, Living Labs are interchangeably used to refer to innovation systems, organisations, user involvement methodologies, real-life monitoring and the European movement (Dutilleul, Birrer, and Mensink). Therefore, Schuurman distinguishes three levels of analysis for Living Labs: an organisational layer, a project layer and an individual user research methods layer (184-185). Co-creation is facilitated at the project layer by introducing a specific Living Lab methodology.

Regarding this methodology, Pierson and Lievens identified the following five consecutive stages in the "process configuration" of a Living Lab project (117) (see Table 1), all of which are tailored towards users and their characteristics. Johansson and Messeter suggest that the process of creating personas should be "a continuous process of reconstructing the user" (240). Therefore, we can state that during every Living Lab research stage we can collect data to enrich personas and their scenarios that will drive the system requirements. The Living Lab methodology serves the purpose of:

- Identifying and modeling personas and their scenarios.

- Refining and validating persona and scenario assumptions based on quantitative and qualitative user data.

- Driving product requirement specification based on personas and scenarios

A main question in the literature that should be answered is: 'How can personas can co-exist with this continuous need for immersion in user data?' Our study suggests Living Lab methodology as a way to structure this process. Each of the five Living Lab project stages aim to develop, validate, and refine the product's target user personas, its key scenarios and product development. Considering Living Lab methodology takes an iterative approach, this will also allow designers to evaluate product requirements in an agile way. 


\begin{tabular}{|c|c|c|c|}
\hline $\begin{array}{l}\text { Consecutive } \\
\text { Living Lab stage }\end{array}$ & What? & $\begin{array}{l}\quad \text { Impact on } \\
\text { Persona } \\
\text { Development }\end{array}$ & $\begin{array}{l}\text { Impact on } \\
\text { Technological } \\
\text { Development }\end{array}$ \\
\hline Contextualizati & $\begin{array}{l}\text { Obtain insights } \\
\text { regarding the product's } \\
\text { background context. } \\
\text { Draw justified selection } \\
\text { criteria and profiles for } \\
\text { the product's intended } \\
\text { end-users. }\end{array}$ & $\begin{array}{l}\qquad \text { Determine } \\
\text { relevant parameters of } \\
\text { the persona and } \\
\text { scenarios such as } \\
\text { identity, goals, behavior, } \\
\text { motivation and system } \\
\text { interaction. }\end{array}$ & $\begin{array}{l}\text { First insights on } \\
\text { the user needs and goals. } \\
\text { Developers can deduce } \\
\text { ways to fulfill these from } \\
\text { the personas and } \\
\text { scenarios. }\end{array}$ \\
\hline Selection & $\begin{array}{l}\text { Identification } \\
\text { and selection of users } \\
\text { that will be involved in } \\
\text { the Living Lab research. }\end{array}$ & $\begin{array}{l}\quad \text { First validation } \\
\text { of persona and scenario } \\
\text { parameters based on } \\
\text { real data by linking them } \\
\text { to foundational } \\
\text { documents which } \\
\text { include user study data } \\
\text { to back up the persona. }\end{array}$ & $\begin{array}{l}\text { This solves the } \\
\text { credibility problem of } \\
\text { the initial developed } \\
\text { personas. }\end{array}$ \\
\hline Concretization & $\begin{array}{l}\text { Modelling specific } \\
\text { characteristics of the } \\
\text { targeted users, such as } \\
\text { their behavior and } \\
\text { perception on the } \\
\text { introduced technology. }\end{array}$ & $\begin{array}{l}\text { Quantitatively refine the } \\
\text { initial persona and } \\
\text { scenario assumptions } \\
\text { using the user cluster } \\
\text { information. }\end{array}$ & $\begin{array}{l}\text { The refined } \\
\text { personas and scenarios } \\
\text { serve as a first interface } \\
\text { between the user study } \\
\text { component and the agile } \\
\text { development process. }\end{array}$ \\
\hline Implementatio & $\begin{array}{l}\text { The actual user } \\
\text { tests }\end{array}$ & $\begin{array}{l}\quad \text { Test users } \\
\text { matching the intended } \\
\text { personas' characteristics } \\
\text { are recruited to use and } \\
\text { evaluate a working } \\
\text { product prototype. }\end{array}$ & $\begin{array}{l}\text { Initial evaluation can be } \\
\text { done on the assumed } \\
\text { requirements }\end{array}$ \\
\hline Feedback & $\begin{array}{l}\qquad \text { An ex-post } \\
\text { measurement, aiming to } \\
\text { detect evolutions in } \\
\text { users' perception and } \\
\text { attitudes towards the } \\
\text { introduced product or } \\
\text { service. }\end{array}$ & $\begin{array}{l}\text { Persona and } \\
\text { scenario evaluation and } \\
\text { optimization }\end{array}$ & $\begin{array}{l}\text { Technological } \\
\text { improvements and } \\
\text { recommendations for } \\
\text { future requirements are } \\
\text { deduced }\end{array}$ \\
\hline
\end{tabular}

Table 1: Living Lab Research Stages (based on Pierson and Lievens),

Persona, and System Development

\section{METHODOLOGY}

Given the exploratory nature of this research, this paper describes an in-depth single case study by means of participatory action research. The purpose of the research was to understand how Living Lab methodology could function as an intermediary between personas and developers by continuous immersion in user data. The case in this study is a European Union funded research project focusing on the enablement of portable and multi-device applications via open-source software. The objective in designing the software was to enable web applications and services to be used and shared consistently and securely over a broad spectrum of converged and connected devices (cross-platform and cross-domain), including mobile, PC, home media (TV) and in-car units. The resulting package, webinosTV, is a 
ubiquitous application for multi-screen media discovery and playback, built to enable a connection between several home devices (e.g. computer, tablet and smartphone) to access and play media files such as movies, music and pictures from any home device on any other device connected to the system.

This case study is interesting because personas were created early in the webinosTV project and no longer used by developers once the first prototype was made. We wanted to investigate whether the effective use of personas during the platform development (upper half of Figure 1) of webinosTV could be raised by introducing the Living Lab methodology and constantly refining and optimizing the personas (lower half of Figure 1).

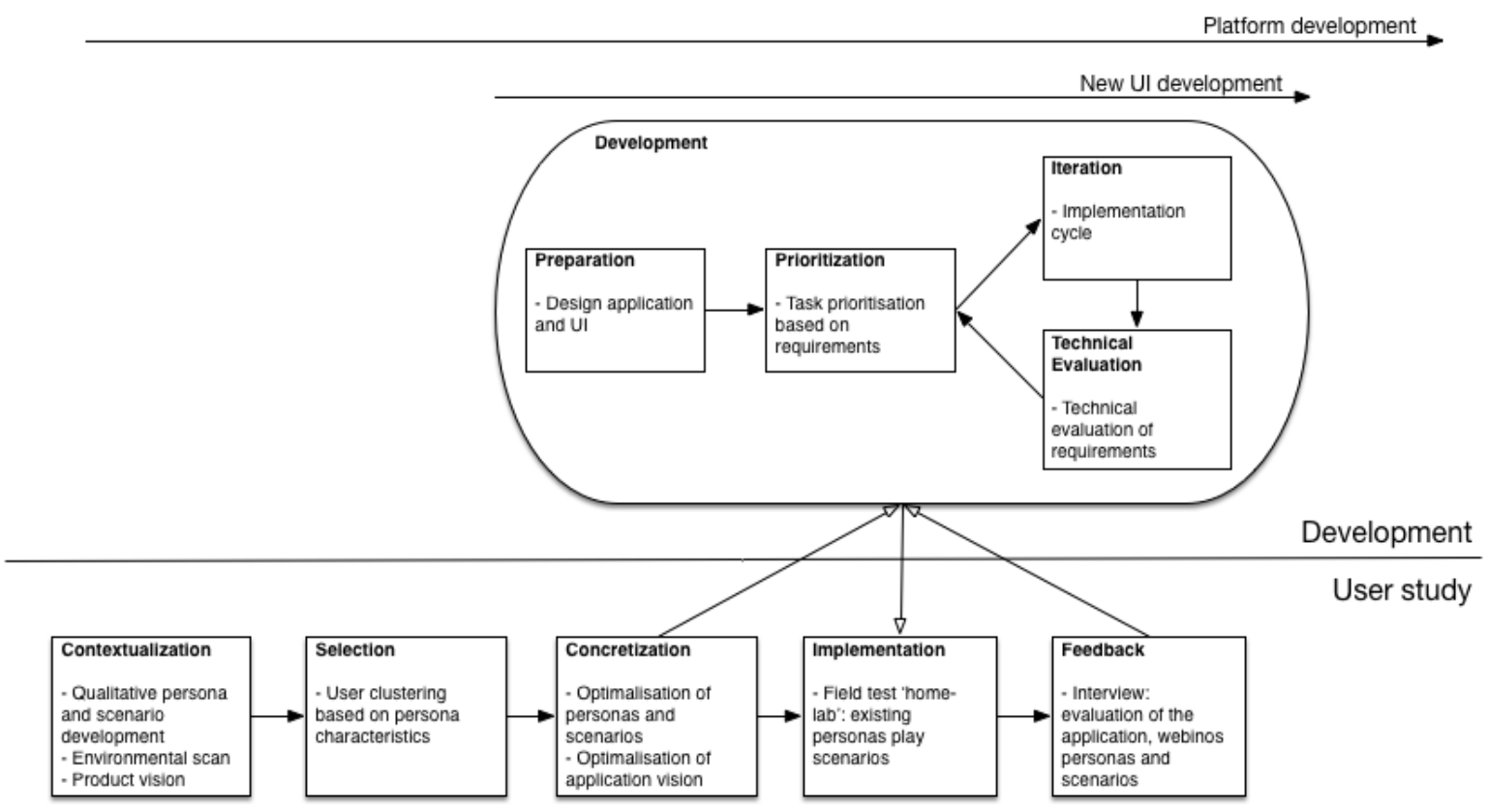

Figure 1: Research Flow

As discussed earlier, the creation of seven personas and their four scenarios occurred prior to before the authors becoming involved in this project. The initial design was completed by academics that approached the technique qualitatively; however, the software developers stopped using the personas after the first prototype was created. To re-engage the design team with the personas, the existing personas were enriched with market data analysed by the authors team during the contextualization phase. Some researchers also suggest combining qualitative and quantitative techniques to improve the effectiveness of personas (McGinn and Kotamraju; Pruitt, Adlin, and Quesenbery). Therefore based on the personas and user scenarios from the first user study phase, the selection phase approaches the problem quantitatively. It does so by using datasets containing real test users' profiles and by clustering this information based on relevant parameters. During the user study's subsequent concretization step, the user cluster information is used to refine the team's initial persona and scenario assumptions. The refined personas and scenarios serve as a first interface between the user study component and the agile development process. Based on these models, the targeted product's key requirements and their assigned priorities are drawn. In turn, the development team can start its agile development iterations in order to deliver a first series of working prototypes, which can be validated in the user study's implementation step (i.e., the fourth Living Lab methodology phase, Figure 1). During this step, test users matching the intended personas' characteristics are recruited to use and evaluate a working product prototype. The testing is performed in a controlled setting. This test setup aims to mimic a domestic and natural 
environment in which the product will eventually be used (e.g., a living room). In a last user study phase, i.e. its feedback phase, interviews are performed with each of the participants, asking for evaluation input and improvement recommendations for the tested product. Moreover, participants are confronted with the previously modeled personas and scenarios and asked to evaluate and/or optimize them. This data is in turn used to qualitatively update the persona and scenario models. The product evaluation results serve as input to the development team via a series of additional product requirements for the user interface.

The application was developed using the Scrum framework (Sutherland) of which every iteration consists of the following steps:

- Preparation: the architecture and user interface of the application are designed

- Prioritization: the issues for both the application and the underlying platform are given a ranking based on their priority.

- Development iteration: This step entails fixing bugs and adding features based on the prioritization done during the previous step. Bugs are fixed based on their priority.

- Technical evaluation: At the end of each sprint, the application and platform are evaluated again, from a technical perspective. To do this, the personas, scenarios and quality attributes that were put forward after each step of the Living Lab methodology are used. All issues encountered are used as input for the prioritization phase of the next sprint.

By following this proposed approach, the persona and scenario development and the immersion in user study data becomes a continuous, iterative process in parallel with prototype development. The data of each step was gathered via extensive monitoring (recordings, realtime observations and field notes), which indicates that the results are mainly based on the author's first experiences of project participation. To complement this, the researchers also interviewed the system developers that were involved in the project. The data was analyzed via open coding and triangulated with the input from the interview.

\section{RESULTS}

\section{Contextualization phase}

As indicated earlier, the webinosTV developers used seven qualitative user personas when working on their first prototype. However, these personas quickly became irrelevant to the design process and we were asked to assist. ${ }^{\text {T }}$ To solve the issues at hand we followed the recommendations of Pruit and Grudin to collect "as much existing research as possible" to enhance credibility (318), especially because the description of the initial personas and the scenarios was very technical. We gathered further insights in users' media consumption behaviour from the digiMeter (iMinds). This yearly survey assembles detailed information on media possession and usage for people living in the Flemish region of Belgium. The study provided some useful insights for the developers such as number of Flemish users multitasking whilst watching TV (87\%) and the type of device they use, namely mobile phone (50\%), laptop (48\%) and tablet (20\%). The data also provided more insights on the digital literacy of Flemish consumers. This enriched the personas and allowed the developers to use them in the creation of a product vision board (See Table 2). 
Product statement: The webinosTV application offers users the possibility to discover media content on any personal computing device and to remotely control this content's playback.

Target group: Media consumers who own one or more personal computing devices such as smartphones, tablets and TV's and want to play media across devices seamlessly. Moreover, there should be no user restrictions based on age, country, etc.

Needs: Users would like to control media playback with just one device (e.g., their phone), so they can playback media on any other device without being in physical proximity (e.g., playing music on the desktop computer while cleaning the room). Moreover, users want to allow friends to share media (e.g., show pictures) on their devices (e.g., the TV in the living room).

Product: webinosTV is a software application, which provides the cross-device; cross-location; cross-user functionality. The app allows for playing back media across devices, e.g. displaying pictures from the smartphone on the TV. Moreover, the app enables rendering media content synchronized on multiple devices at the same time. Each device can act as a media source; playback target; or remote controller.

Value: Using webinosTV results in less frustration when trying to get media playback working across devices and locations. It works across all types of devices. It allows you to play media on other devices seamlessly, and doesn't require any physical connection. No more fussing about with incompatible devices. Moreover, the app is not restricted to one user. Hence, webinosTV makes it easy to allow friends to use your devices for media playback.

Competitors and alternatives: Airplay by Apple: proprietary solution only works with Apple products. Other vendor-specific combinations: again not fully cross-device. Using USBsticks or other media storage cards: requires hooking devices up physically. UPnP/DLNA: limited set of supported devices and setting up cross-location access support is cumbersome and frustrating.

Table 2: Product Vision Board: Multi-device Media Consumption Application

\section{Selection}

To increase the personas' accuracy (=representativeness) even more, it is recommended to "couple user research and persona development tighter" by using quantitative techniques (Sinha 1). Therefore we performed a Principal Component Analysis ii . We used the most relevant variables of the digiMeter study, which also occur in the application's persona and scenario assumptions and the product's vision board (with KMO = $0.87, \mathrm{p}<0.001$ ). Three matching factors were found: the variety of media usage (crohnbach's $\alpha=0.90$ ), the frequency of media usage (crohnbach's $\alpha=0.60$ ) and the user's multi-device usage (crohnbach's $\alpha=0.90$ ). Based on a comparison of these primary factors with the application's assumption personas and scenarios, these deemed consistent factors for clustering. Hierarchical clustering suggested a three-cluster solution and they were established with a K-Means clustering algorithm. Each cluster covers a distinct user type, which we named: basic user, technology addict, and pleasure seeker (see Table 3). 


\begin{tabular}{|l|l|l|l|}
\hline & $\begin{array}{l}\text { Basic user } \\
(\mathbf{N = 1 2 6 0})\end{array}$ & $\begin{array}{l}\text { Technology } \\
\text { addict }(\mathbf{N = 5 2 6})\end{array}$ & $\begin{array}{l}\text { Pleasure seeker } \\
(\mathbf{N = 8 7 0})\end{array}$ \\
\hline $\begin{array}{l}\text { Media usage } \\
\text { frequency }\end{array}$ & 2.60 & 3.92 & 4.03 \\
\hline $\begin{array}{l}\text { Media variety } \\
\text { usage }\end{array}$ & 2.37 & 5.85 & 5.04 \\
\hline $\begin{array}{l}\text { Multi-device } \\
\text { usage }\end{array}$ & 0.18 & 5.05 & 0.14 \\
\hline
\end{tabular}

Table 3: Clusters deduced from digiMeter database (factors mean matching score)

To make the link between the persona and the supporting data explicit, we created a "foundational document" (Grudin and Pruitt 319). During a meeting with the developers, relevant data points and facts were linked to the personas. The following picture depicts an example of how the results of the clustering were communicated to the developers:

\section{TECHNOLOGY ADDICT}

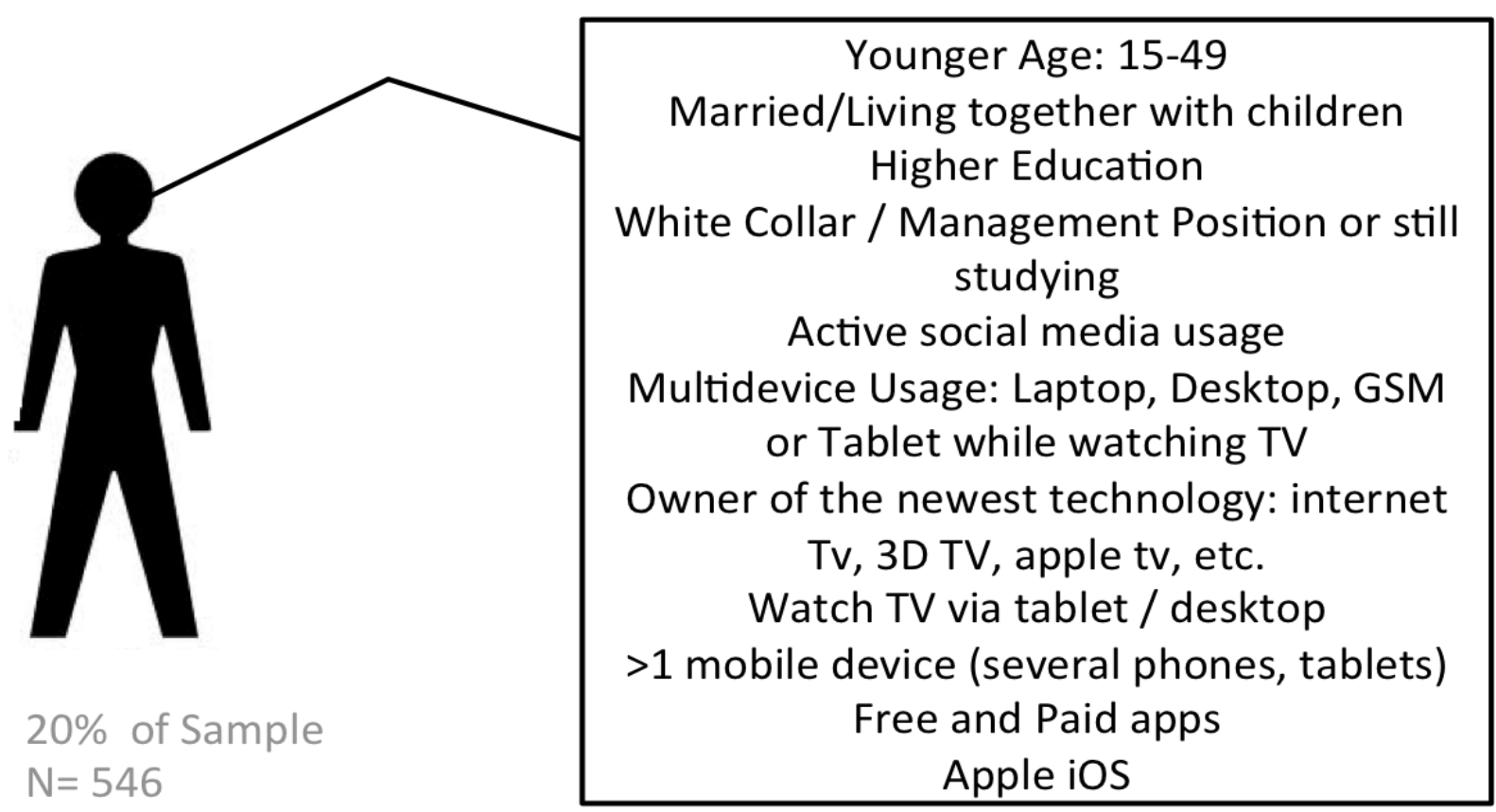

Figure 2: Cluster information as communicated with development team

\section{Concretization}

Based on the quantitative user characteristics extracted from the three clusters, the qualitative persona assumptions defined in the contextualization phase were extended and refined. The scenarios of the different personas were completely rewritten based on the possibilities of the webinosTV application as well as the input from the digiMeter data.

The description of Georg for example was expanded tremendously. His aptitudes, motivations and privacy concerns were extended towards his desire for comfort and the level of experience that new technologies offer him. Multitasking, time saving and using whichever 
device is closest to him defines Georg's behaviour in every step of the way. Following picture shows how Georg was represented in a persona poster to the developers:

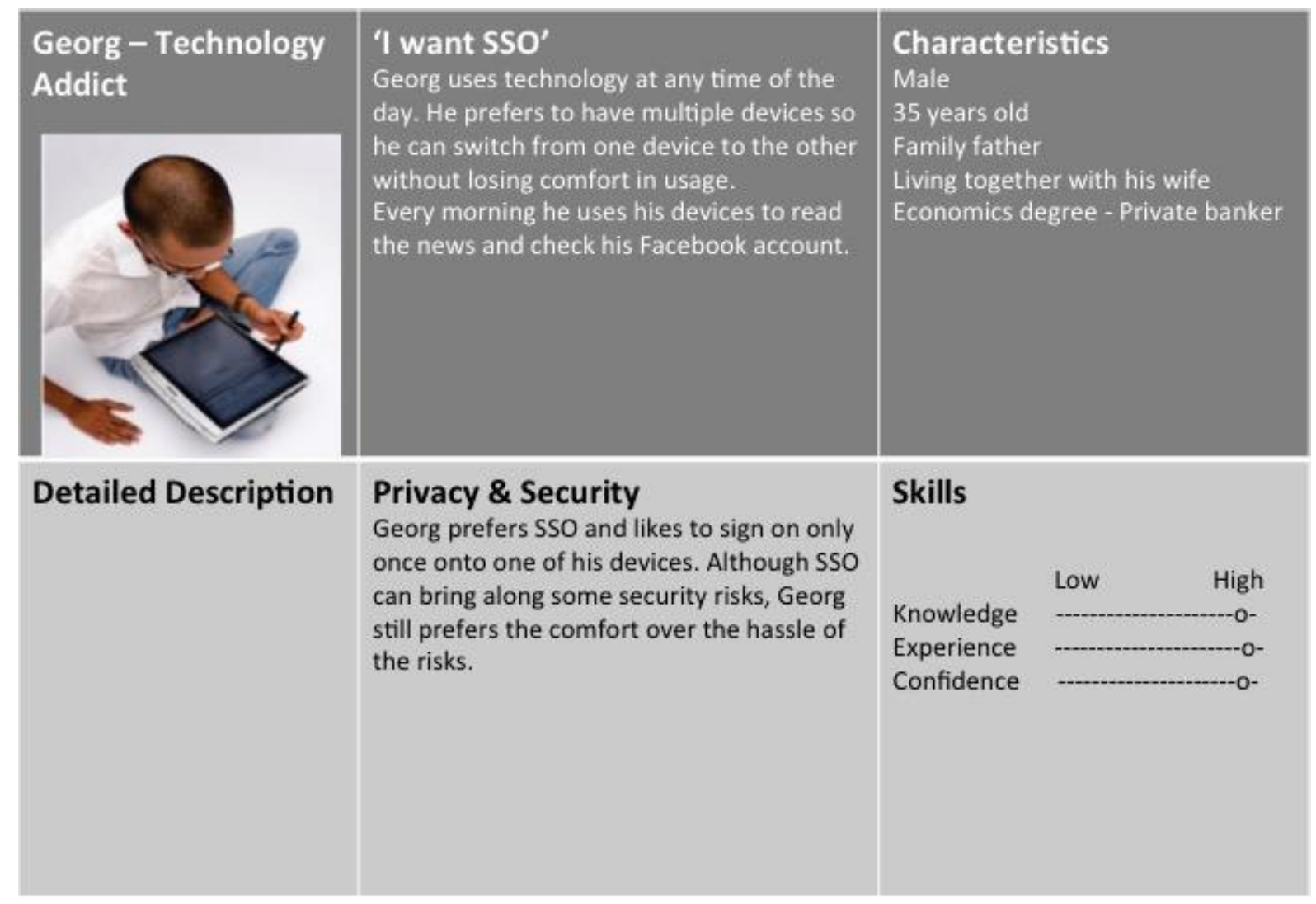

Figure 3: Poster Version of Persona 'Georg'111

There also was a text foreseen in the foundational document explaining the persona and the accompanying scenario of Georg more in depth. The scenario looked as following:

Watching a movie on the TV, while streaming it from smartphone/tablet

Overview: Georg wants to show a movie taken from his phone to a group of friends.

Description: Georg's weeks are filled with client visits, meetings and travel. It is Friday evening and some friends are coming over he haven't seen in a while. They sit on the sofa and talk about what has changed in their lives during the past couple of weeks. They relax with a drink, some finger food, and get comfortable. All of the sudden, Georg wants to show some videos of his crazy pet and its behaviour during the last couple of weeks. He takes out his tablet/phone and streams the movie to the TV. Afterwards, one of his friends takes out his phone and shares a recent video he shot during the performance of his favorite artist. It doesn't take long before everybody is sharing content.

Issues: Georg wants to show a video to all of his friends.

Benefits: Webinos provides an easy to browse system, where you can search your content via different devices. The overview you get, makes it easy to choose the content you want.

Usability Breakdown: Persona - Georg; Duration - Minutes; Frequency - Monthly; Demands - Low; Goal Conflict - Low. 
The refined personas and application scenarios from the selection and concretization phase were used to drive the agile development of webinosTV. The product's main requirements and priorities (i.e., product backlog) were set based on the knowledge acquired from the user study. Because the personas have varied technical skills, and users expect applications to "just work", the user experience needed to be optimized. In the preparation phase, there were several problems that needed to be fixed:

- The layout was based on a purely left-to-right workflow

- On mobile, there was no general overview, so the user did not grasp the application's workflow.

- The concept of distributed media playback was new to most users and was something they didn't understand without an additional explanation.

Based on the outlined problems, some general interface design patterns were discussed and chosen. The prototype resulting from this phase looks as following:

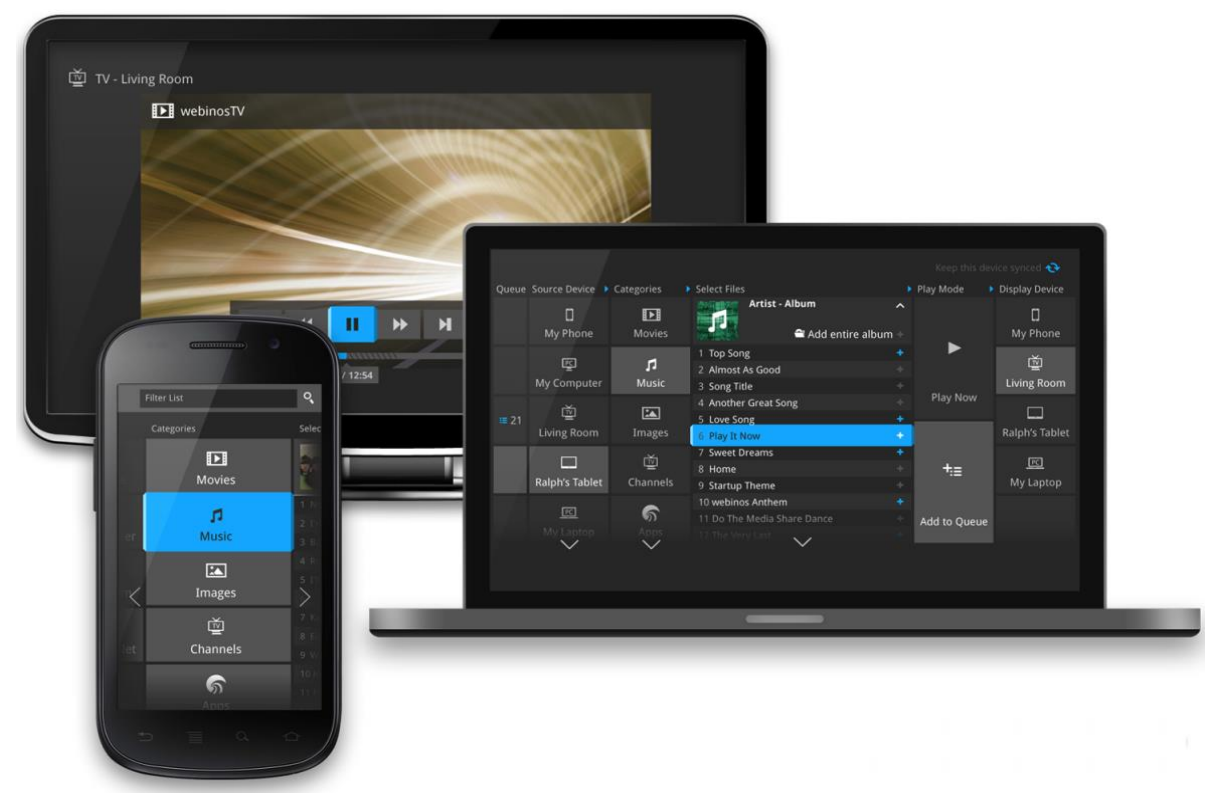

Figure 4: Testable Prototype

The identified issues were prioritized and the main drivers for the scrum calls were threefold. First, the user interface was redesigned and the interface for tablet and mobile was developed separately from the interface for TV. Second, the persona and scenario concretization served as input for the user interface redesign, and for the evaluation and testing rounds that happened before every scrum call. By using the refined personas and scenarios as input, testing from a user perspective was made a lot easier, thus providing more concrete feedback. Third, stability and performance testing was carried out as preparation for the actual user testing. The output of these tests was given back to the platform development team, accelerating the stabilization of the platform.

\section{Implementation}

Cluster characteristics of the previous steps predicted pleasure seekers and technology addicts to be the main innovators and early adopters of the platform. Therefore the remainder of the study we focused on these two groups. All test users were selected from the digiMeter 
database. During eight test sessions, a total of 18 users evaluated webinosTV. Eight users were recruited based on their characteristics matching either the pleasure seeker $(n=4)$ or technology addict $(n=4)$ personas. Each of these users was asked to invite one or two friends to jointly try out and evaluate the product.

The eight test sessions were held at a lab reflecting a living room. Living Lab methodology focuses on testing with "real users" reflecting "real daily use" (Sauer 48). The 'real' dimension of the users being present during the test is obvious, but the 'real daily life' dimension is open for discussion. Living Lab methodology suggests a 'real daily life' environment to learn from the "unexpected ideas of users and their unexpected interactions with the technology" (Sauer 24). To align with this vision, we provided the users with a minimal introduction to webinosTV's concepts. Moreover, the matching personas' pre-modeled application scenarios were suggested as a potential starting point to familiarise with the product. Nevertheless, after the introduction, users were given complete liberty to use the application however they wanted. By giving them this freedom and letting them bring a friend, we tried to replicate a 'real daily life' dimension to uncover unexpected events as would be possible in a non-lab environment. All participants were observed during the entire test session. This already distinctively confirmed previous persona assumptions from the digiMeter clustering. Test subjects with characteristics matching the Georg and Justin personas (i.e., technology addicts), immediately explored the product and did not follow the suggested application scenario. The majority of these users only enacted the proposed scenario at the very end of the testing session, as they first tried out all available functionalities. This observation confirms the assumption that technology addicts are multifunctional users when it comes to technology and they like variation in their usage patterns. Compared to other users, technology addicts appeared faster at grasping the concepts of webinosTV's multi-device technology. Additionally, technology addicts were observed to be more self-centered when interacting with a technological product. These users tend to test out the product without involving or interacting with other people (i.e., the friends they invited to the session). The technology addicts, e.g., regularly interfered with other users' playlists by overriding their actions and individually selecting another movie or song for playback.

The test was also organized to identify potential usability problems with webinosTV. Usability refers to the ease of using a new application. Six quality attributes were researched during the usability testing:

- Learnability: How easy is it for users to accomplish basic tasks the first time they encounter the design?

- Efficiency: Once users have learned the design, how quickly can they perform tasks?

- Memorability: When users return to the design after a period of not using it, how easily can they work with the application the next time?

- Errors: What and how many errors do users make, how severe are these errors, and how easily can they recover from the errors?

- Satisfaction: How pleasant is it to use the application and its design?

- Utility: Does the application provide the user with the wanted features?

Although the designers tested the application in advance by using the personas and scenarios as guided test scripts, the test phase indicated the platform was not as intuitive as initially anticipated. Most participants had difficulties getting familiarized with webinosTV's multidevice functionality. The observations during the testing phase showed users tend to get into a virtual cocoon with the personal computing device in front of them. Due to this single-device focus, many external stimuli were ignored. Users often did not notice actions on one device 
triggering an event on a remote device. For example, based on the devices' configuration, clicking the "play media" button might result in a remote device starting the actual playback. Although the remote media playback was always clearly audible and/or visible, these events did often go unnoticed. For some participants, it took a moment to grasp this core concept. Nevertheless, once they had the hang of the application, all users started enjoying it and were visibly amazed by its potential. These results indicate that personas and usability tests are complementary and can enhance the outcomes for designers. The developers received new perspectives on the user logic flow of the application, providing them with extra input to optimize the users experience design.

\section{Feedback}

Each testing session was concluded with a feedback interview. Supported by an interviewer, the test users were asked about their experience and the usability of the application. Two additional questions were asked in order to obtain feedback concerning webinosTV's assumption personas and application scenarios. After being presented with these models, the test users are asked the following questions: Q1: Would you expect these people to actually use the application? Why (not)? Which characteristics would you change? Do you recognize yourself in any description? Q2: Would you expect these scenarios to be typical user stories for webinosTV? Why (not)? How would you adapt them to better map to the targeted users? Would you use webinosTV similarly to the described scenarios? Why (not)? How would you use it?

Based on these interviews, a SWOT analysis to improve different requirements was compiled, along with a final qualitative refinement of the key user personas and their application scenarios. A SWOT analysis (strengths, weaknesses, opportunities, and threats) consists of four evaluation categories and aims to identify both the internal and external factors that influence a particular objective (both positive as well as negative influences). The strengths and weaknesses aspects focus on internal characteristics. The opportunities and threats, on the other hand, aim to identify and evaluate externally originating elements.

Strengths.

- One access point - multiple device connection (comfort)

- Speed of actions

- Common playlist

- Ability to use it outside your own network

- Content sharing: e.g. work

- Fun: entertainment and social

Weaknesses.

- Usability: Interface too complex and not intuitive

- Limited media options (only 15 items in playlist possible)

- No option to choose content in playlist (ranking, changing, selecting, shuffle, etc.)

- Look and feel

- Too many clicks to perform actions

Opportunities.

- Expand potential: third party content such as Spotify access

- Extra content options: e.g. create your own music quiz

- Collaboration options e.g. making a playlist together 
Threats.

- Competitors integrate more: e.g. AppleTV

- Fast changing technology market

- Technological dependencies

When looking at the overall usability of the application, it appears most (70\%) target groups experience difficulties with the learnability of the application and problems the first time they performed basic tasks. Once they knew how the design worked, they were satisfied with the speed of their performed actions (100\%).

The memorability aspect was evaluated poorly. Especially in regard to the older users, the participants were convinced they would have to explain the actions over and over again because it is perceived as being too complex. The main error made by the users was not clicking on the plus button to add items to the queue of the playlist. This is a major issue, because without this action they cannot succeed their goals, namely playing media files. Almost none $(90 \%)$ of the users were capable of solving this error on their own without input from the developers. All the other errors users made were minor and easy to solve.

After the evaluation of the application, participants were also asked to assess the different personas based on their potential usage of the application and the contexts in which they would use it. This provided us with the opportunity to further optimize webinosTV's modeled personas and application scenarios. Particularly the application scenarios did require major changes, while the input on the user personas was rather limited. The users also recognized themselves in the persona they were matched to. Georg for example required a minor update in the need for quality time with his daughter in the persona description, which impacted the suggested scenario towards using the application with his daughter instead of his friends.

Scenario: Watching a movie on the TV, while streaming it from smartphone/tablet

Overview: Georg wants to show a movie to his child after she is finished doing her homework.

Description: Georg's weeks are filled with client visits, meetings and travelling from one place to another. Once home he helps his daughter with her homework. As a reward for finishing up her homework, she can watch a movie. Georges has several movies on his computer and grabs the device closest to him to select the movie she can watch. His daughter seems not satisfied with the selected movie and requests a new one. Georges browses his laptop and decides to show another one she seems in the mood for.

Issues: Georg wants to show a video to his daughter. With the ability to select from a wider variety of suitable catalogue entries

Benefits: webinosTV provides an easy to browse system where you can search your content via different devices. The overview you get, makes it easy to choose the content you want stored on any device connected.

Usability Breakdown: Persona - Georg; Duration - Minutes; Frequency - Daily; Demands - Low; Goal Conflict - Low 
The findings of the implementation and evaluation allowed the agile development team to drive the end-of-iteration validation of their potentially shippable product. The developers primarily focused on the weaknesses of the SWOT to enhance user experience of webinosTV. Initially the user logic flow (observed during the test) of the application was optimized to create a more stable and user-friendly version of webinosTV. The requirements coming from the revised scenarios served as input for future improvements of webinosTV.

\section{DiscUSSION}

The principle aim of this study was to assess whether using the Living Lab methodology could provide structure and improve the effective use and impact of personas in the product development process by merging user data with personas. The results indicate that there are positive benefits associated with this approach in a design project. Personas strengthen the focus on the end user, their tasks, goals and motivation. The continuous (re)construction of personas served as a constant reminder for the developers of for whom they were creating the platform. The merit of this approach lies in the combination of personas with user study data: this makes the personas less abstract, the underlying data is seen as trustworthy, and the resulting information is perceived as relevant to the design process. The input from the quantitative study, for example, provided the developers with additional test scenarios and made them aware of the fact that their application was not intuitive enough for 'regular users'. This in turn made them revise the user interface of the application.

User experience researchers recognize the difficulty of predicting future use contexts without any previous product experience. This is also reflected in the adjustments made to the scenarios over time. By bringing personas 'to life', and inviting designers to participate in a semi-real-life test, new understandings of the market and domain specific knowledge can be brought to light. This 'real life' testing provides insights into the usefulness and usability of an application which is not possible with fictional characters such as personas. These additional insights on user experience then provide the developers with new assessments of necessary requirements to ensure market acceptance (for example, raise the number of items in the playlist queue, or guide the user away from the single device focus). In other words, personas can communicate user needs without additional user study data in the initial stages of the project, but a combination of both improves the decision making throughout the entire development process. By combining these two approaches, personas are no longer purely used as a tool for communication, but also help to determine priorities in design requirements or test scripts.

Table four provides a framework, based on the Living Lab methodology, to continuously develop personas in combination with user study data, and demonstrates the added value realized for developers at each stage in the project. The table indicates that a one off creation of personas and their scenarios only has limited effect. Therefore it is important to continuously develop personas and scenarios quantitatively and qualitatively. Living Lab methodology allows personas to become a dynamic given, evolving through the different research steps and bringing them to life for the developers. If all the data being gathered is delivered to the development team, it can serve as feedback for adjusting the requirements and priorities for future iterations. This in turn enhances the overall quality of the design. 


\begin{tabular}{|l|l|l|}
\hline Consecutive stage & $\begin{array}{l}\text { Persona \& Scenario } \\
\text { Refinement }\end{array}$ & Added Value for Developer \\
\hline Contextualization & $\begin{array}{l}\text { Initial persona and scenario } \\
\text { enrichment with additional } \\
\text { market data }\end{array}$ & $\begin{array}{l}\text { Need information } \\
\text { Ability to develop Vision Board }\end{array}$ \\
\hline Selection & $\begin{array}{l}\text { Validation of existing personas } \\
\text { and scenarios via clustering }\end{array}$ & $\begin{array}{l}\text { Prioritization of features that will be } \\
\text { developed in the prototype based on } \\
\text { input. This in turn drove the team to } \\
\text { better meet internal deadlines. }\end{array}$ \\
\hline Concretization & $\begin{array}{l}\text { Making personas and } \\
\text { scenarios more accurate with a } \\
\text { focus on: } \\
\text { Identity } \\
\text { Roles } \\
\text { Motivations } \\
\text { test scripts }\end{array}$ \\
\hline Implemactions & $\begin{array}{l}\text { Bringing personas and } \\
\text { scenarios to life }\end{array}$ & $\begin{array}{l}\text { Solution and evaluative information: } \\
\text { usability input, optimization of } \\
\text { functional, personal and social } \\
\text { requirements }\end{array}$ \\
\hline Feedback & $\begin{array}{l}\text { Optimization of scenarios for } \\
\text { future usage }\end{array}$ & $\begin{array}{l}\text { Solution and Evaluative information: } \\
\text { input on usability, personal, social } \\
\text { and contextual requirements }\end{array}$ \\
\hline
\end{tabular}

Table 4: Consecutive research steps, personas and added value for development

The empathy towards the personas increased over time, and in turn this allowed the developers to focus more on the end-user during all the stages of the development process. The developers also confirmed that because the real users selected their associated persona as 'the one' matching their own characteristics, this made the personas more 'real' and 'vivid' for developers, which boosted their belief in the added value of personas as a tool to develop. This study shows that the Living Lab methodology structures the triangulation of user data with the personas and their accompanying scenarios. As such, more realistic and accurate assessments of users are created and their impact on design in every step of the process is noticeable for developers. By bringing the personas to life via the Living Lab approach, more detailed design solutions can be deduced because the 'real' personas provide input on 'real' scenarios. This provides more input on requirements that will influence the product's use in the field and user experience can be optimized accordingly. Overall, the results show that this approach prevents developers from perceiving users purely as objects that have to fit in their design and emphasizes the user, its needs and potential interactions with the design. During stability tests for example, the personas and their scenarios allowed the developers to think about new test scenarios and find extra bugs in the system. Introducing 'real' users' to the design allowed the developers to observe 'real interactions' such as overriding someone's playlist and provided them with feedback to prioritize the next steps in the development process.

Although these results favour a Living Lab methodology to bridge the gap between personas, users, their data and the development team, one should not underestimate the resources needed to implement this technique. These resources include expertise in preparing the usability test, having access to a lab in which the tests can be performed, and incentives for potential users to partake in the test. Therefore this approach might not be suited for every 
development process, and in particular budgetary constraints may render the use of this method invalid. Personas are sometimes used as an alternative method when Living Lab methodology is not possible. In such cases, the results of our study may not apply. Yet despite these limitations, we want to emphasize the importance of the Living Lab methodology to increase product acceptance. Not combining persona and scenario development with user studies can have severe effects on the development process.

In this single case study the scenarios changed over time, and even towards the end of the project they were still undergoing change. If users were not confronted with the technology and asked to think about scenarios of use, the developers might have imagined an incorrect scenario when developing extra features for the platform, leading to a product that only has limited market acceptance. Additionally, the input from the testing phase on user experience and usability allowed the developers to create a more intuitive and stable product.

This study also has limitations beyond those of the methodology explored above. The results are based on a single case study and although the triangulation of the data should control for the impact of the context, the results can only be seen as an indication and not be generalized to wider populations. Future research should try to replicate and validate the results of this study in order to make the findings more generalizable. Replication provides some challenges because most user experience researchers will not have access to a panel of potential participants and a database to deduce characteristics of personas.

\section{CONCLUSION}

This article presented a framework to enhance the effective use of personas via interactive coupled open innovation, and optimize user experience accordingly. We have demonstrated in a single case study that users' assumption personas and their scenarios can be iteratively validated and refined, and as such increase efficiency of the development process. Despite personas being advocated for design and communication activities, their usage in practice is often limited. Even in the webinosTV case the personas were initially only used to discover needs. By introducing the Living Lab methodology linking persona development and user data to the development process, the effectiveness of the personas was improved. The Living Lab methodology complements the initial developer-based UX point of view by introducing new unaddressed user experience focal points into the scope of the development process, which otherwise go unnoticed. Quantitatively and qualitatively refining personas and their scenarios drove the key product requirements and priorities. The methodology allows designers to focus on prioritization in the development process allowing them to gain time and tailor the product towards 'real' future use scenarios. For webinosTV, assumption personas were quantitatively clustered and refined via the Digimeter profile database. The refined personas were used for driving the application's agile development iterations. Moreover, 18 test users and their friends, recruited from the digiMeter database by matching the targeted personas, validated the delivered potentially shippable products. User tests were performed in a lab aiming to mimic a domestic environment. Evaluation results were gathered through observations and interviews with test participants. Their input was used for assessing the tested product's strengths, weaknesses, threats, and opportunities (SWOT analysis). Participants were asked to qualitatively evaluate the application's assumption personas and user scenarios. By bringing the personas to life in this phase the developers were able to create a viable product with an optimized user experience design. 


\section{END NOTES}

i For a more elaborate description of these initial persona and application scenario assumptions, we refer the interested reader to the deliverable in reference list (Paul).

ii For more information about the use of PCA we advise you to consult Jolliffe.

iiii Image Robin 2008: http://blog.litcentral.com/wp-content/uploads/image/tablet\%20 man.jpg

\section{WORKS CITED}

Chapman, C. N., and R. P. Milham. “The Personas' New Clothes: Methodological and Practical Arguments against a Popular Method." Proceedings of the Human Factors and Ergonomics Society Annual Meeting 50.5 (2006): 634-636. Web.

Coenen, Tanguy, and Sarie Robijt. "Towards FALL: A Framework for Agile Living Lab Projects." 2 (2015): n. pag. Print.

Dotan, Amir et al. "Designing with Only Four People in Mind? - A Case Study of Using Personas to Redesign a Work-Integrated Learning Support System." HCI. N.p., 2009. 497-509. Web.

Dutilleul, Benoît, Frans a J Birrer, and Wouter Mensink. "Unpacking European Living Labs : Analysing Innovation 'S Social Dimensions." Central European Journal of Public Policy 4 (2010): 60-85. Web.

Eriksson, Mats, Veli-pekka Niitamo, and Seija Kulkki. State-of-the-Art in Utilizing Living Labs Approach to User- Centric ICT Innovation - a European Approach. Sweden: N.p., 2005. Print.

Floyd, Ingbert R., M. Cameron Jones, and Michael B. Twidale. "Resolving Incommensurable Debates: A Preliminary Identification of Persona Kinds, Attributes, and Characteristics." Artifact 2.1 (2008): 12-26. Web.

Fowler, Martin, and Jim Highsmith. "The Agile Manifesto." Software Development 9.August (2001): 28-35. Web.

Grudin, Jonathan, and John Pruitt. "Personas . Participatory Design and Product Development: An Infrastructure for Engagement." Proceedings of the Participatory Design Conference. Palo Alto: N.p., 2002. 144-152. Web.

iMinds. digiMeter: Adoption and Usage of Media \& ICT in Flanders. N.p., 2013. Print.

Johansson, Martin, and Jörn Messeter. "Present-Ing the User: Constructing the Persona." Digital Creativity 16.4 (2005): 231-243. Web.

Jolliffe, Ian. Principal component analysis. John Wiley \& Sons, Ltd, 2002.

Long, Frank. "Real or Imaginary: The Effectiveness of Using Personas in Product Design." Proceedings of the Irish Ergonomics Society Annual Conference. N.p., 2009. 1-10. Print.

Markensten, E., and H. Artman. "Procuring a Usable System Using Unemployed Personas." ACM International Conference Proceeding Series. Vol. 82. N.p., 2004. 13-22. Web.

Matthews, Tara, Tejinder Judge, and Steve Whittaker. "How Do Designers and User Experience Professional Actually Perceive and Use Personas?" Conference of Human Factors in Computing Systems. Austin, Texas: N.p., 2012.1219-1228. Web.

McGinn, Jennifer Jen, and Nalini Kotamraju. “Data-Driven Persona Development.” Proceeding of the twentysixth annual CHI conference on Human factors in computing systems CHI 08 (2008): 1521. Web. CHI'08.

Paul, A. Updates on Scenario and Use Cases. N.p., 2012. Web.

Pierson, Jo, and Bram Lievens. "Configuring Living Labs for a 'Thick' Understanding of Innovation." Ethnographic Praxis in Industry Conference. Vol. 1. Redmond, WA: N.p., 2005. 114-127. Print.

Piller, Frank, and Joel West. "Firms, Users, and Innovation: An Interactive Model of Coupled Open Innovation." New Frontiers in Open Innovation. N.p., 2014. 1-33. Print. 
Pruitt, John, and Jonathan Grudin. "Personas: Theory and Practice." Proceedings of the 2003 Conference on Designing for User Experiences - DUX '03. N.p., 2003. 1. Web.

Pruitt, John S., Tamara Adlin, and Whitney Quesenbery. The Persona Lifecycle. N.p., 2006. Web.

Sanders, Elizabeth B, and Pieter Jan Stappers. "Co-Creation and the New Landscapes of Design." CoDesign 4.1 (2008): 5-18. Print.

Sauer, Sabrina. "User Innovativeness in Living Laboratories: Everyday User Improvisations with ICTs as a Source of Innovation." Universiteit Twente, 2013. Web.

Schuurman, Dimitri. "Bridging the Gap between Open and User Innovation? Exploring the Value of Living Labs as a Means to Structure User Contribution and Manage Distributed Innovation." UGent/VUB, 2015. Print.

Sein, Maung K et al. "Action Design Research.” MIS Quarterly 35 (2011): 37-56. Print.

Sinha, Rashmi. "Persona Development for Information-Rich Domains." CHI '03: CHI '03 extended abstracts on Human factors in computing systems (2003): 830-831. Web.

Sutherland, Jeff. Scrum Handbook. N.p., 2010. Web.

Trimi, Silvana, and Jasmina Berbegal-Mirabent. "Business Model Innovation in Entrepreneurship." International Entrepreneurship and Management Journal 8.4 (2012): 449-465. Web. 11 Nov. 2013. 